\title{
Characterization and Psychophysical Studies of an Air-Jet Lump Display
}

\author{
James C. Gwilliam, Student Member, IEEE, Matteo Bianchi, Member, IEEE, \\ Lester K. Su, and Allison M. Okamura, Fellow, IEEE
}

\begin{abstract}
Development of tactile displays to enhance palpation of lumps during robot-assisted minimally invasive surgery is challenging due to size and weight constraints, motivating a pneumatic actuation strategy. This work describes the quantitative and psychophysical assessment of an air-jet tactile display that creates a lump percept by directing pressurized air through an aperture onto the finger. The air pressure and aperture size are meant to control the hardness and size, respectively, of the perceived lump. Jet impingement pressure and flow rate were measured by capacitive tactile sensors and mass flow meters at varying aperture sizes and pressures. The air-jet pressure profile width evolves as jet theory predicts and is largely independent of supply pressure (and therefore jet exit velocity). The method of constant stimuli was used to determine the just noticeable differences (JNDs) for the air pressure and aperture size. Qualitative results indicate that subjects perceive the stimulus as a "lump-like" shape. Pressure JNDs ranged from 19.6$24.4 \mathrm{kPag}$ and aperture size JNDs ranged from 0.50-0.66 mm. No significant correlation exists between the supply pressure and changes in perceived lump size. However, pressure JNDs show significant $(p<0.001)$ inverse correlation with aperture size, with improved discrimination at larger apertures, where a greater finger pad area is stimulated.
\end{abstract}

Index Terms-Haptics, RMIS, tactile display, lump display

\section{INTRODUCTION}

$\mathrm{R}$ OBOT-ASSISTED minimally invasive surgery (RMIS) systems have augmented traditional minimally invasive surgery (MIS) by providing surgeons with stereoscopic vision, increased dexterity and precision, and motion scaling. In such systems, the surgeon interacts with the tissue through teleoperation, which isolates the surgeon from direct physical contact with the tissue. Kinesthetic (force) and tactile (cutaneous) feedback are minimal, leaving surgeons to rely almost exclusively on visual cues [1].

Much of the previous haptic feedback research for MIS and RMIS applications has focused on kinesthetic feedback, e.g., [2], [3], [4]. The need to restore tactile feedback during many surgical tasks remains a well-documented challenge [5]. One such task is lump detection, which requires localizing hard lumps embedded in the soft tissues of the body. Cancer, for example, typically manifests as hard lumps (tumors) in the soft tissues of the breast, prostate, lung, and other tissues. These lumps are significantly stiffer than the surrounding tissue [6], [7] and are embedded beneath the tissue, and therefore not visible. These lumps can be localized during open surgery when the surgeon's

- J.C. Gwilliam is with the Department of Biomedical Engineering, The Johns Hopkins University, Baltimore, MD 21210.

E-mail: jim.gwilliam@jhu.edu.

- M. Bianchi is with the Interdepartmental Research Center "E. Piaggio," Faculty of Engineering, University of Pisa, Largo Lucio Lazzarino 1, Pisa 56122, Italy. E-mail: matteo.bianchi@centropiaggio.unipi.it.

- L.K. Su and A.M. Okamura are with the Department of Mechanical Engineering, Stanford University, Stanford, CA 94305.

E-mail: \{lester.su,aokamura\}@stanford.edu.

Manuscript received 25 May 2012; revised 25 May 2012; accepted 26 Oct. 2012; published online 1 Nov. 2012.

Recommended for acceptance by S. Choi.

For information on obtaining reprints of this article, please send e-mail to: toh@computer.org, and reference IEEECS Log Number TH-2012-05-0038.

Digital Object Identifier no. 10.1109/ToH.2012.71. fingertips are in direct contact with the tissue and distributed tactile information is naturally available. In minimally invasive procedures, insufficient tactile feedback makes lump localization and assessment extremely challenging [8] and in some cases can greatly limit the surgeon's ability to stage the disease [9].

Tactile displays have been proposed to provide tactile feedback to a surgeon. Perhaps the most common type of existing tactile display is the "pin-array" tactile display, driven by a range of actuation technologies, including shape memory alloys [10], [11] and piezoelectrics [12]. Although pin-array displays can recreate a broad range of stimuli upon the finger pad, their bulk [13] and complex electromechanical designs do not satisfy the requirements for implementation on a robot-assisted surgical system; namely, a practical system must occupy a small footprint (for integration on a master-side manipulator), and not impede the motion or dexterity of the master manipulators. Few studies have demonstrated tactile displays capable of integration within an RMIS system [14], [15].

The size and weight constraints of RMIS have driven the development of novel pneumatically actuated displays, which do not require a mechanical assembly at the point of contact with the user's skin and therefore typically operate with less hardware (and size) than other tactile displays. Existing pneumatic displays use arrays of air-filled tubes to push a membrane [16], balloons [17], [18], or pins [19], [20] directly against the finger. Although the valves to control such devices are typically located upstream from the display, significant tubing is required to control each display "element" of the array individually.

A very different approach was proposed by Inoue et al. [21], in which a single nozzle directs a high-flow air jet onto a flexible membrane beneath the finger pad. Here, the term 


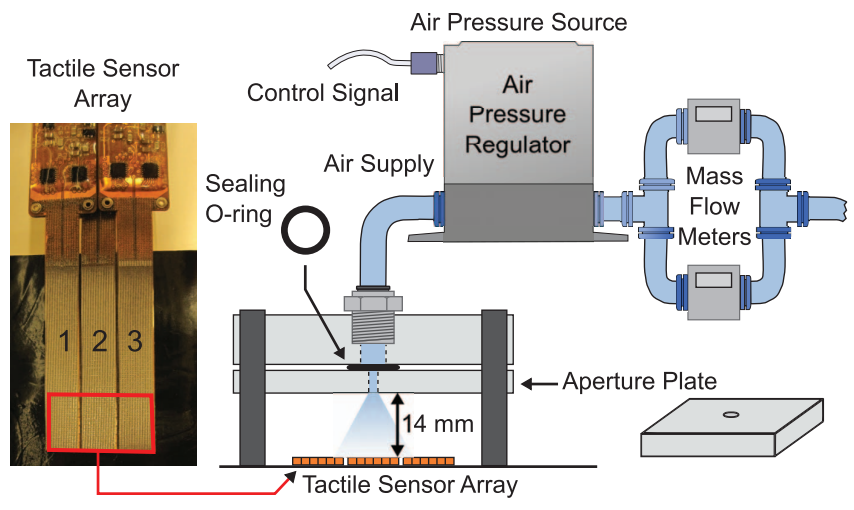

Fig. 1. Setup for tactile sensor and air flow measurements. The air pressure regulator allows pressurized air through the aperture plate and controls supply pressure while mass flow meters record flow rate. An array of capacitive tactile sensors centered under the aperture records distributed pressure as supply pressure and aperture size are varied.

"air jet" describes air flowing from a nozzle over some distance until it impinges on the membrane. The authors demonstrate that a user can feel a lump due to the pressure on the membrane, and the size of the lump can be controlled by the distance between the nozzle and the membrane. One disadvantage of this approach is that the entire membrane is displaced by the air jet, not just the local lump area.

We propose a pneumatic air-jet approach in which pressurized air impinges directly on the finger through a small circular aperture, creating a hemispherical "lump-like" indentation in the finger pad. The display has two control parameters: 1) the supply pressure of the air, and 2) the size of the aperture through which the air escapes. These two variables, controlled independently, are meant to control the perceived hardness and size of the lump, respectively.

An air jet that impinges directly on the skin, like the one described in this work, evokes a sensation that mimics feeling a lump directly, as opposed to feeling a lump embedded in tissue. Depending on the application, it may be desirable to display lumps directly to enhance sensation. (Alternatively, a membrane approach may be preferred due to its potential for realism in an application such as surgical simulation.) The direct air-jet approach remains largely uncharacterized as a haptic stimulus both quantitatively and psychophysically. Previous psychophysical research for pneumatic stimuli is largely limited to the aforementioned non-air-jet type display mechanisms, including studies on pneumatic tactile cues [16] and evaluations of pneumatic tactile displays [18], [20]. In this work, we characterize the direct air-jet stimulus through several means, including 1) an analytical model based on fluid dynamics, 2) a quantitative study measuring the display output directly using capacitive tactile sensors, and 3) perceptual experiments to determine the smallest changes in supply pressure and aperture size that are detectable to the human finger.

\section{Design and Characterization}

\subsection{Lump Display and Measurement Apparatus}

The lump display and measurement apparatus consist of four main components:
TABLE 1

Parameters for Tactile Sensor Measurements

\begin{tabular}{|c|c|c|c|c|c|c|}
\hline \multicolumn{7}{|c|}{ Aperture Diameters (mm) } \\
\hline D1 & D2 & D3 & D4 & D5 & D6 & D7 \\
\hline 1.0 & 1.9 & 2.3 & 2.75 & 3.2 & 3.6 & 4.5 \\
\hline \multicolumn{7}{|c|}{ Supply Pressures (psig) } \\
\hline P1 & P2 & P3 & $\mathbf{P 4}$ & P5 & P6 & P7 \\
\hline 10 & 20 & 30 & 40 & 50 & 60 & 70 \\
\hline
\end{tabular}

1. an electronically controlled air pressure regulator,

2. a set of interchangeable acrylic "aperture plates," each with a different aperture diameter,

3. a tactile sensor array to measure the resulting impingement pressure, and

4. mass flow meters to measure the air flow rates in the system.

Together these components yield an apparatus for which aperture size, exit velocity, and impingement pressure of a single air jet can be controlled and measured (Fig. 1).

The electronically controlled pneumatic regulator (SMCITV2031-21N2L4, SMC Corporation, Noblesville, IN) provides step-less control of air pressure (0.05-0.5 MPa) proportional to an electrical signal $(0-5 \mathrm{~V})$, with a maximum flow rate of $1,500 \mathrm{~L} / \mathrm{min}$. Linearity between the input signal and set pressure is within \pm 1 percent full scale (F.S.), hysteresis within \pm 0.5 percent F.S., and sensitivity within 0.2 percent F.S. Two mass flow meters (C100L-DD-3-0V1SV1-PVIC-V1-SO-CO-GS, Sierra Instruments, Monterey, CA) were placed in parallel upstream of the pressure regulator to record flow rates.

The aperture size was set manually using one of seven distinct acrylic aperture plates (5.4 $\mathrm{mm}$ thick) ranging from $1.0-4.5 \mathrm{~mm}$ in aperture diameter (Table 1). A single aperture plate was clamped to the underside of the apparatus with the aperture centered over the air source, separated by a rubber O-ring to create an airtight seal. The regulatorsupplied air was forced through the aperture and directed onto the surface of the tactile sensor array.

The tactile sensor array is composed of three adjacent smaller sensors (DigiTacts, Pressure Profile Systems, Los Angeles, CA) each containing 24 sensing elements, that together form a $6 \times 12$ array of tactile sensing elements, occupying a total footprint of $12 \times 25 \mathrm{~mm}$ (Fig. 1 (left)). The sensors are capacitive and provide a linear response with a sensing range of $0-0.14 \mathrm{~N} / \mathrm{mm}^{2}$ and a sensitivity of $6.9 \times$ $10^{-4} \mathrm{~N} / \mathrm{mm}^{2}$. The sensor array was centered $14 \mathrm{~mm}$ beneath the aperture.

\subsection{Air-Jet Theory}

The air-jet lump display can be described using standard fluid mechanics models. A jet is defined as a system in which a fluid (e.g., air) issues unidirectionally from a localized source into a quiescent fluid. With the downstream movement of the fluid, the jet spreads laterally, and its peak velocity decreases, as the initial jet fluid momentum diffuses conservatively into the ambient fluid.

The nature of the momentum diffusion is governed by the jet Reynolds number, which can be defined as 


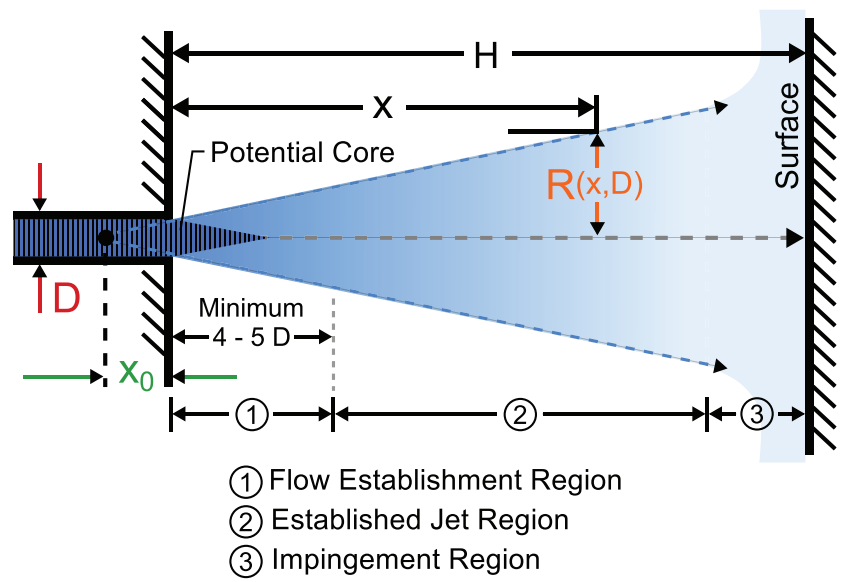

Fig. 2. An ideal round jet develops as a conical shape with a jet spread rate, $S$. The jet radius $(R)$ is a function of the distance from the outlet $(x)$ and the aperture diameter $(D)$. The jet exhibits properties that can be divided into three distinct regions of interest.

$R e=U_{0} D / \nu$, where $U_{0}$ is the jet speed at the exit, $D$ is the exit diameter, and $\nu$ is the kinematic viscosity of the jet fluid. Here, we estimate $U_{0}$ as the jet bulk velocity, $Q / A$, where $Q$ is the volumetric flow rate of the jet fluid, which we measure explicitly, and $A$ is the nozzle cross-sectional area. For low Re (relatively low jet momentum), the flow is laminar, and diffusion is primarily viscous. For high $R e$ (high jet momentum), the flow is turbulent, and diffusion is driven by the random, turbulent transport of fluid parcels at small scales. In the present work, Re ranges from 0.25$1.25 \times 10^{5}$, indicating that the jet is turbulent.

The impinging turbulent jet flow here evolves through three distinct regions (Fig. 2). The "flow establishment" region immediately downstream of the jet nozzle (1), which includes the "potential core," is where the turbulent jet properties initially develop and there remain regions of the jet fluid that are unmixed with the surrounding fluid. In the "developed" region (2), the turbulent jet shows a selfsimilar form for the radial profile of the axial velocity, and also exhibits asymptotic scaling for jet spread and decay of centerline velocity [22]. In particular, the jet spreads linearly with the axial distance from the nozzle, $x$, which can be written as

$$
\delta_{\frac{1}{2}}(x)=S\left(x-x_{0}\right),
$$

where the jet width, $\delta_{\frac{1}{2}}(x)$, is the radial distance from the centerline to the point where the axial velocity profile drops to half its maximum value, and $x_{o}$ is the virtual origin.

The jet maximum axial velocity (which occurs on the centerline) decays inversely with $x$ as

$$
\frac{U_{C}(x)}{U_{O}}=B\left(\frac{x-x_{0}}{D}\right)^{-1},
$$

where $U_{C}(x)$ is the jet centerline velocity at a distance $x$ from the jet nozzle, and $B$ is an empirical constant called the velocity decay coefficient [23]. Finally, in the impingement region (3), the flow is altered by the presence of the plate. One can reasonably approximate the pressure on the plate using inviscid flow theory. In particular, the peak pressure occurs on the jet centerline, where the mean velocity at the plate is zero.

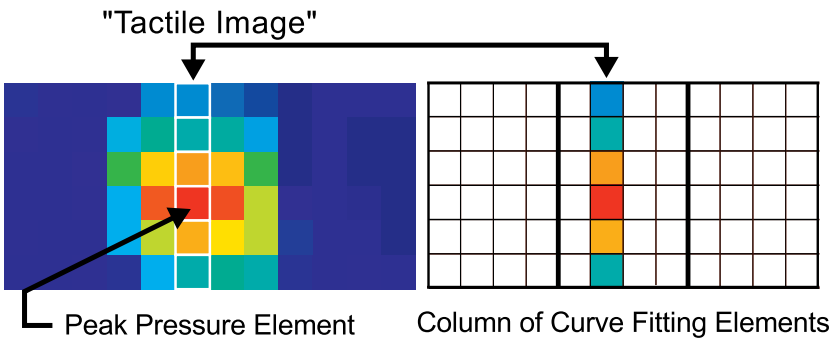

Fig. 3. Tactile data terminology. (Left) Sensor element data were averaged, resulting in a "tactile image" with a peak pressure value. (Right) The peak pressure element determines which column of sensor element values is used for the pressure profile curve fit.

Some of the present run conditions, particularly for the smallest aperture sizes, show supersonic jet bulk velocities. This complicates mixing properties immediately outside the jet exit by introducing compressibility effects. We assume here that entrainment of ambient air allows us to neglect the effects of compressibility at the downstream location of the tactile array.

The model described here serves primarily to define the expected behavior of the air jet in terms of flow, velocity, and rate of jet expansion, depending on the region of jet development. The quantitative results (Section 2.4) reveal that the jet exhibits no unexplainable properties and behaves according to jet theory, which gives confidence that the subsequent psychophysical experiments were performed with a known, well-characterized jet.

\subsection{Quantitative Characterization}

\subsubsection{Measurement}

To determine the complex relationships between the air supply pressure, the aperture size, and the resulting jet velocity and impingement pressure, tactile sensors measured impingement pressure using every combination of seven aperture sizes and seven supply pressures (Table 1). A tactile sensor measurement occurred at $35 \mathrm{~Hz}$ for $30 \mathrm{~s}$, after which recording stopped and the sensor was zeroed with a new baseline. For each aperture size, measurements began with the smallest selected supply pressure (10 psig) and proceeded in ascending order followed by descending order of supply pressure measurements. Afterward, two mass flow meters measured the flow rate under the same experimental conditions.

\subsubsection{Analysis}

A "tactile image" was formed from each measurement by averaging sensor element values across the duration of the measurement. Each resulting tactile image was evaluated in terms of the peak pressure value (Fig. 3 (left)) and the distributed pressure profile. The column of tactile image values containing the peak pressure value were used to analyze the resulting pressure profile distribution (Fig. 3 (right)). In all cases, the peak pressure element and column are expected to be near the center of the tactile sensor array since the air jet was centered over the sensors. Although using a column of sensor elements provides fewer points for curve fitting, it ensures that the elements used span a single sensor, thus eliminating intersensor variability. Measuring the display output in this manner allows for 


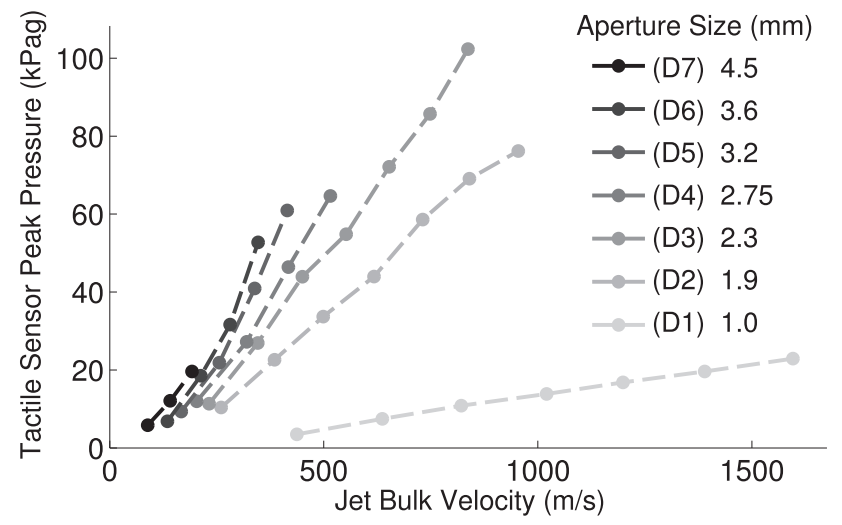

Fig. 4. Peak pressure values obtained from tactile sensor plotted against calculated jet bulk velocity. Each line represents a single aperture size. Traces with fewer points contained peak pressures that were not attainable at the specified aperture size.

comparison between empirically measured results and those predicted by the jet theory model ((1) and (2)). Additionally, the quantitative results are required for establishing the desired pressures for the perceptual experiments.

\subsection{Tactile Sensor Results}

\subsubsection{Tactile Image Peak Pressure}

Fig. 4 shows the results for the tactile sensor peak pressure as a function of jet exit velocity. Each line represents a specific aperture diameter, $D$. As expected, the sensor peak pressure correlates positively with the jet exit velocity for each value of $D$. However, the data show that as $D$ decreases, the sensor peak pressure for a given jet exit velocity also decreases. The sensitivity to aperture size is particularly strong for the smaller $D$ values $(D 1-D 4)$. This is likely due to the fixed distance, $H$, between the jet exit and the sensor array, which means that the dimensionless impingement ratio, $H / D$, increases as $D$ decreases. For larger $H / D$, the jet momentum undergoes greater diffusion into the ambient air, resulting in lower local flow velocities and thus reducing the peak pressures at the sensor surface.

\subsubsection{Tactile Image Gaussian Fitting}

Fig. 5 shows the pressure profiles obtained from the column of tactile sensor elements containing the peak pressure value. Since the static pressure profile of an air jet at an impinging surface is approximately Gaussian [24], a twodimensional Gaussian curve was fitted to these averaged pressure profiles (Fig. 3) using an unconstrained nonlinear minimization of the sum of squared residuals. The Gaussian curve is written

$$
y=\alpha e^{\frac{-(x-\mu)^{2}}{2 \sigma^{2}}},
$$

where $\alpha$ is the amplitude of the curve, $\mu$ represents the centering of the curve with respect to the tactile sensor array, and $\sigma$ defines the spatial spread, or standard deviation, of the curve. Each individual plot in Fig. 5 represents the pressure profile and curve fit for a specific aperture size and supply pressure. Plots with shaded backgrounds indicate measurements for which the specified supply pressure was not completely attainable. In general,

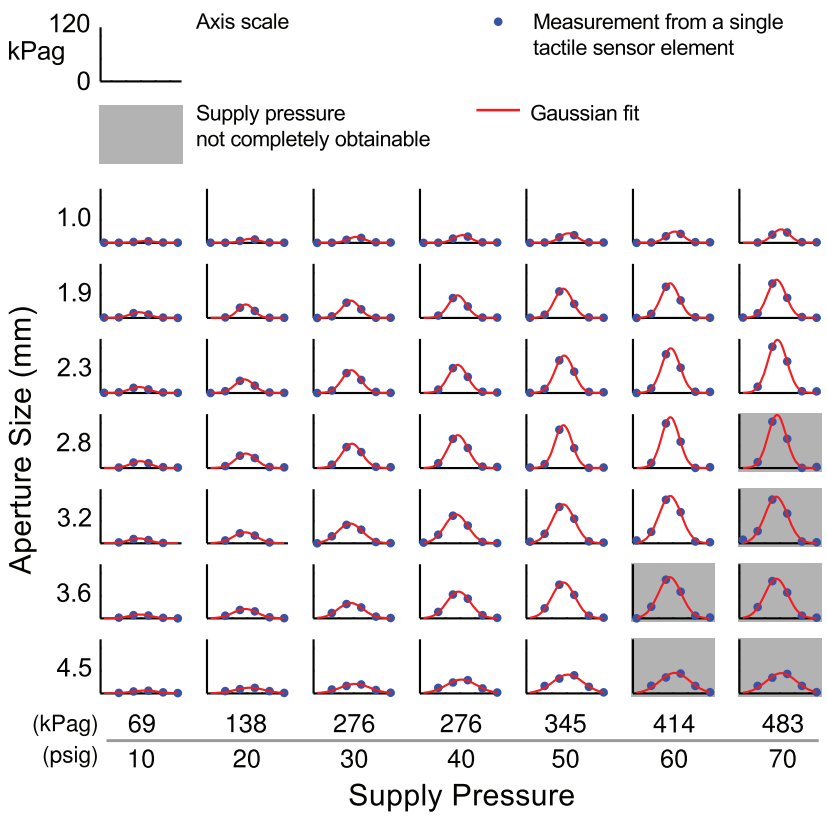

Fig. 5. Gaussian curve fits for each tactile measurement. The curve is fit to the profile of the column of tactile sensor elements containing the peak pressure value.

the magnitude of the curve (and the total sensor pressure force) increases as the supply pressure increases, as expected. To quantitatively assess the change in pressure profile shape as a function of the stimulus parameters, we evaluated the Gaussian parameters over the span of supply pressures and aperture sizes used in the study.

Fig. 6a shows the amplitude $(\alpha)$ of the Gaussian curve fits. Fig. $6 \mathrm{~b}$ shows the spatial spread $(\sigma)$ of each Gaussian fit. Two important observations can be made from these data: First, $\sigma$ increases monotonically as aperture size increases, with few exceptions. Second, for a single aperture size, $\sigma$ is largely independent of the supply pressure. Fig. 6c shows $r^{2}$ values for each fit, indicating how closely the Gaussian curves fit the tactile sensor pressure profile data. Results indicate that as aperture size increases, $r^{2}$ values decrease rapidly, especially for smaller supply pressures. This may be explained by two factors: First, when the downstream impingement distance $(H)$ is less than 4-5 times as large as the aperture diameter $(D)$, the impingement occurs in the potential core of the flow establishment region, where the flow profile has not yet become Gaussian [22]. Second, at lower supply pressures, the sensor signal-to-noise ratio is at a minimum, allowing the sensor noise to dominate the prescribed fit of the curve.

The Gaussian term $\mu$ in (3) describes the offset distance between the center of the Gaussian fit with respect to the center of the tactile sensor array. This term might physically represent the position error during placement of the aperture over sensor array. In all cases, this error was within $\pm 0.5 \mathrm{~mm}$.

Fig. 7 shows Gaussian fits for supply pressure P4 (40 psig). Plot ordinates are nondimensionalized by $\alpha$ (left) and by the area under each Gaussian curve, $A_{c}$ (right). Upper row plots show actual position on the abscissae, while lower plot abscissae are nondimensionalized by $\sigma$.

The width of a Gaussian curve described by (3) at a height of $\alpha / 2$ is referred to as the full-width 


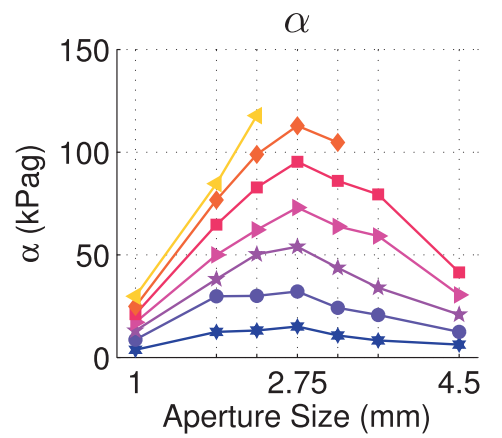

(a)

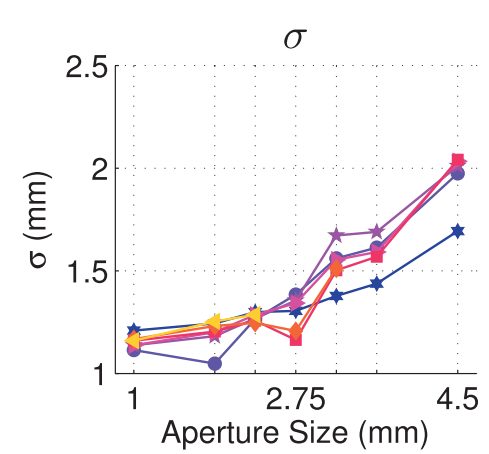

(b)

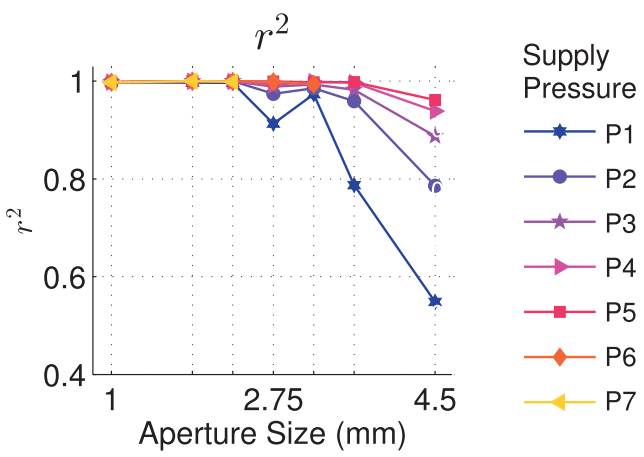

(c)

Fig. 6. Gaussian fit parameters and $r^{2}$ values for all aperture sizes and supply pressures. (a) Gaussian fit amplitudes ( $\alpha$ ) show similar responses to peak pressure values. (b) Gaussian fit variance $(\sigma)$ shows consistent increases in spatial spread as aperture size increases. For a single aperture, $\sigma$ is largely independent of supply pressure. (c) Coefficient of determination $\left(r^{2}\right)$ values show how each Gaussian curve fits to corresponding tactile sensor pressure profile.

at half-maximum, and is denoted here by $2 b$. Fig. 8 shows the nondimensionalized variation of $2 b / H$. For larger $H / D, b$ is proportional to $H$ (linear fit, $R^{2}=0.99$ ), indicating a linear rate of jet expansion with increasing distance from the source, as the jet theory model predicts. For $H / D \leq 5$ (i.e., for impingements of the potential core occurring in the flow establishment region), $2 b / D$ approaches a value of 1 . Fig. 8 also shows data from $\mathrm{Tu}$ et al. [24] acquired from a plane jet over a slightly larger range of $H / D$, which compares favorably with the measured data of the air-jet lump display.

\section{Perceptual Experiments}

\subsection{Subjects}

All participants in this study were right-handed volunteers and gave informed consent to perform psychophysical
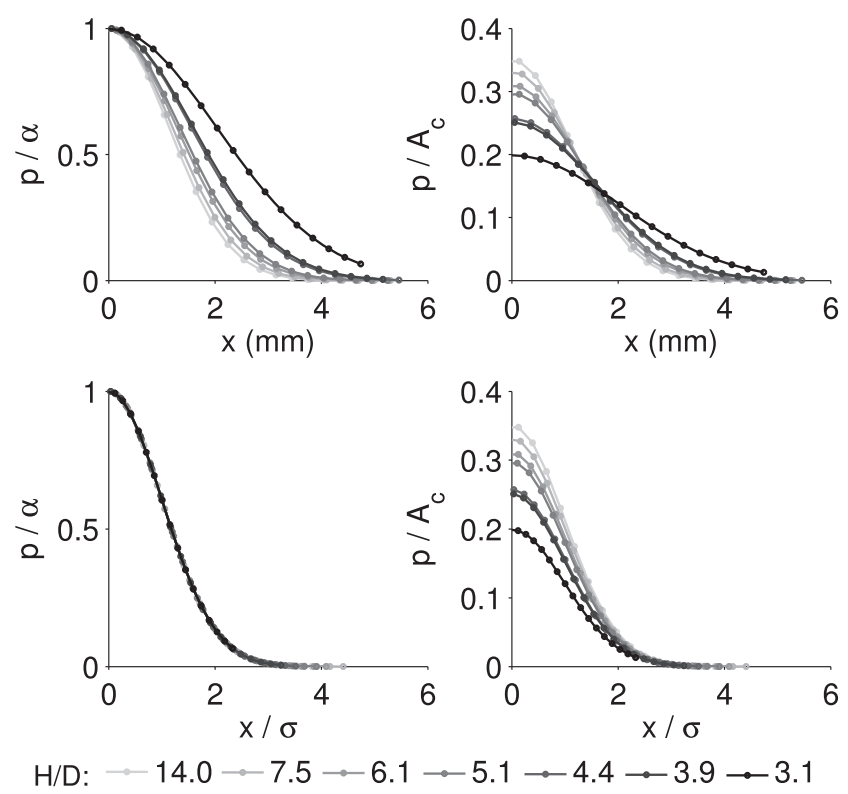

Fig. 7. Gaussian fits for supply pressure P4 (40 psig). Abscissae are normalized by $\alpha$ (left column), and area under the curve, $A_{c}$ (right column). Ordinates are nonnormalized (top row) and normalized by $\sigma$ (bottom row). Each curve is centered manually by amount $\mu . H / D$ is the dimensionless impingement ratio. experiments. No subjects reported physical limitations that would affect their ability to perform the experiment tasks. All data collected in this study were approved by The Johns Hopkins University Homewood Institutional Review Board. The three perceptual experiments performed in this study are summarized in Table 2.

\subsection{Common Setup}

Subjects were seated with the right index finger fixed in a finger holder, palm down, with the center of the distal finger pad exposed and centered over the aperture (Fig. 10 (left)). A skin-safe adhesive tape (Trutape LLC) was applied along the back of the index finger spanning from the fingernail to the base of the finger to restrict joint movement about the interphalangeal joints. The distance from the exposed finger pad to the aperture was fixed at $14 \mathrm{~mm}$, consistent with the distance used in the tactile sensor experiment.

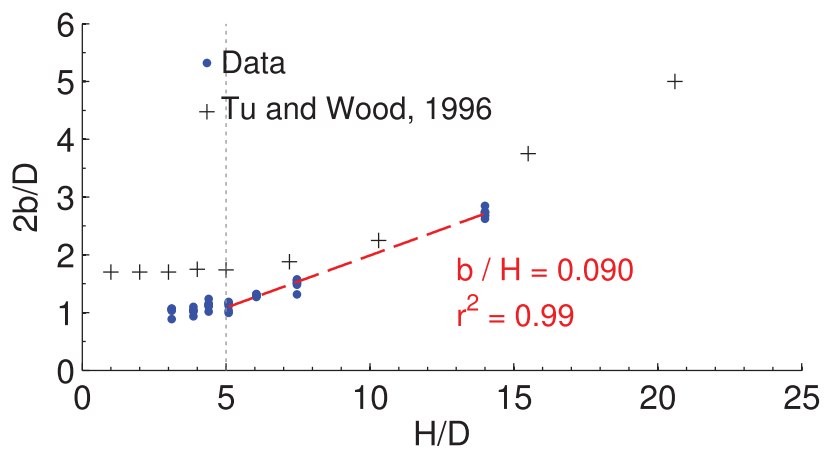

Fig. 8. Dependence of pressure profile width on $H / D$. For approximately $H / D>5, b$ is proportional to $D\left(r^{2}=0.99\right)$. For approximately $H / D \leq 5$ (i.e, for impingement of the potential core), $2 b / D$ is nearly constant with a value approaching 1 .

TABLE 2

Summary of Perceptual Experiments

\begin{tabular}{lcccc}
\hline Experiment & Males & Females & Age Range & Section \\
\hline Open Response & 6 & 4 & $20-30$ & 3.3 \\
Pressure JND & 5 & 5 & $20-28$ & 3.5 \\
Size JND & 11 & 5 & $20-32$ & 3.6 \\
\hline
\end{tabular}


TABLE 3

Stimuli for Open Response Paradigm Experiment

\begin{tabular}{|c|c|c|}
\hline \multirow[b]{2}{*}{ Stimuli } & \multicolumn{2}{|c|}{ Aperture Size (mm) } \\
\hline & $\underset{(1.6 \mathrm{~mm})}{\mathbf{A}}$ & $\begin{array}{c}\text { B } \\
(2.5 \mathrm{~mm})\end{array}$ \\
\hline Peak Pressure (kPag) & \multicolumn{2}{|c|}{ Supply Pressure (psig) } \\
\hline P1 20 (kPag) & 20 & 14 \\
\hline P2 60 (kPag) & 62 & 36 \\
\hline
\end{tabular}

\subsection{Open Response Paradigm Experiment}

\subsubsection{Experimental Task}

We conducted a preliminary perceptual experiment to evaluate how effectively the air-jet display could produce a compelling lump percept. Subjects were provided with four different air-jet stimuli $\left\{A_{P 1}, A_{P 2}, B_{P 1}, B_{P 2}\right\}$, summarized in Table 3 . Supply pressures required to produce the desired peak pressures are also indicated.

The experiment administrator alternated the stimulus between the two aperture sizes $(A$ or $B)$ and the two pressure stimulus levels $(P 1$ or $P 2)$ as verbally requested by the subject. At all times, subjects could view the current stimulus parameters on a computer display. Subjects were allowed to experience each stimulus as many times as desired (each stimulus lasted 1.5 seconds), including small lateral movements. After subjects deemed their exploration of the four experiment stimuli sufficient, they were asked to draw on paper the profile of the shape they perceived during interaction with each stimulus.

\subsubsection{Results}

Eight of the ten subjects drew lump-like shapes for all four stimuli, similar to those drawn by subject 5 (Fig. 9a). Subject 1 drew a rectangular shape for stimulus $B_{P 1}$ but with rounded corners (Fig. 9b), while Subject 10 drew a sharp triangular profile for stimulus $B_{P 2}$ and a wide rectangular profile for stimulus $B_{P 1}$ (Fig. 9c). The results suggest that the majority of subjects perceived profiles that were curved in shape, many of them resembling a Gaussian curve. Equally important, most subjects correctly varied their depictions of the profile amplitude and width between the four different stimuli, suggesting that they perceived changes in the applied pressure level and pressure profile width, which are important for displaying lumps of different perceived hardness and size, respectively. The subsequent psychophysical experiments in this study investigated the smallest increments in stimulus parameters required for subjects to notice differences in lump profile perception.

\subsection{Psychophysical Method and Analysis}

We conducted two psychophysical experiments to determine the just noticeable difference (JND) for both supply pressure (Section 3.5) and aperture size (Section 3.6). The psychophysical procedure and analysis common to both experiments are described in this section.

\subsubsection{Psychophysical Method}

We used the psychophysical method of constant stimuli and followed a two-alternative forced choice protocol in which subjects received pairs of distinct stimuli to the finger pad. Each stimulus pair consisted of a reference stimulus (RS) and a comparison stimulus (CS), always presented in random order. Subjects were asked to indicate which stimulus produced the larger pressure percept (JND pressure experiment) or had a perceived larger contact area upon the finger pad (JND aperture size experiment). A single trial consisted of the first stimulus (1.5 s), an interstimulus interval (ISI), and the second stimulus (1.5 s), followed by the subject's response. An external monitor displayed " 1 " or " 2 " during the presentation of the first or second stimulus, respectively, followed by "Respond Now" at the conclusion of stimulus pair. Subjects submitted their response ("1" or "2") using an external numerical keypad, with their responses being stored and written to a file. Subjects were permitted to experience a particular stimulus pair as many times as necessary by pressing any other key, which would repeat the previous stimulus pair. For both experiments, the comparison stimuli were equally spaced about the RS. The minimum and maximum CS were chosen in a preliminary study such that they were almost

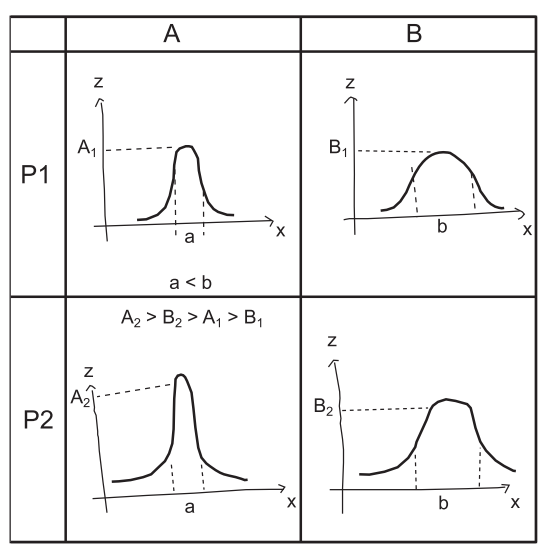

(a) Typical Response (Subject 5)

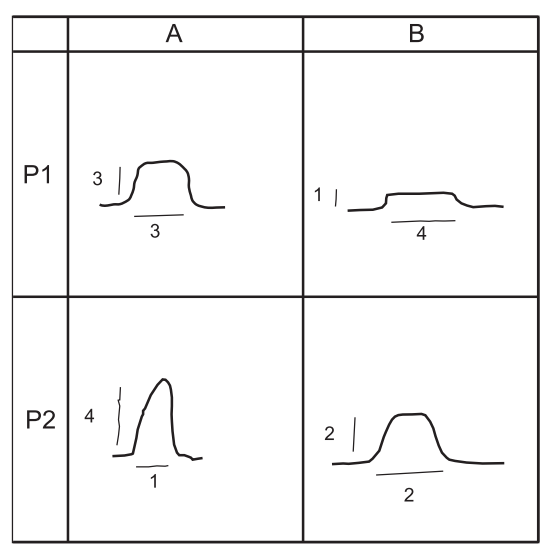

(b) Subject 1: Round Corners

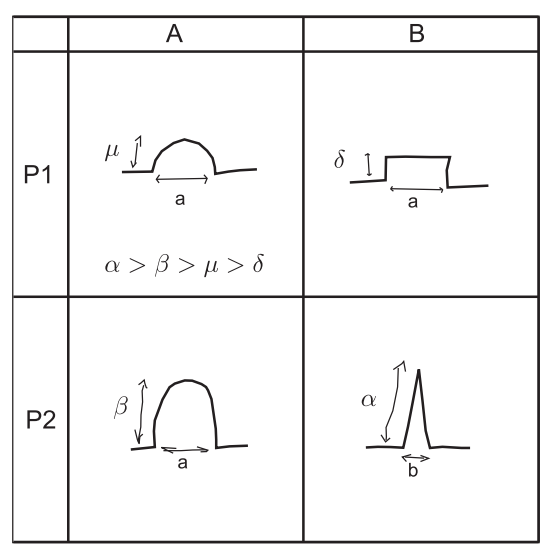

(c) Subject 10: Sharp Corners

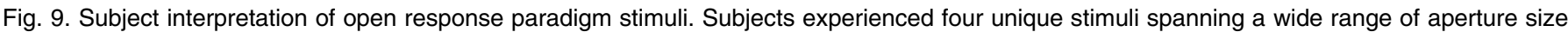

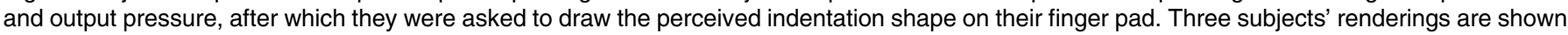
here for each of the four stimuli. Alphanumeric characters used by subjects to denote scaling are shown in typeset to improve clarity. 


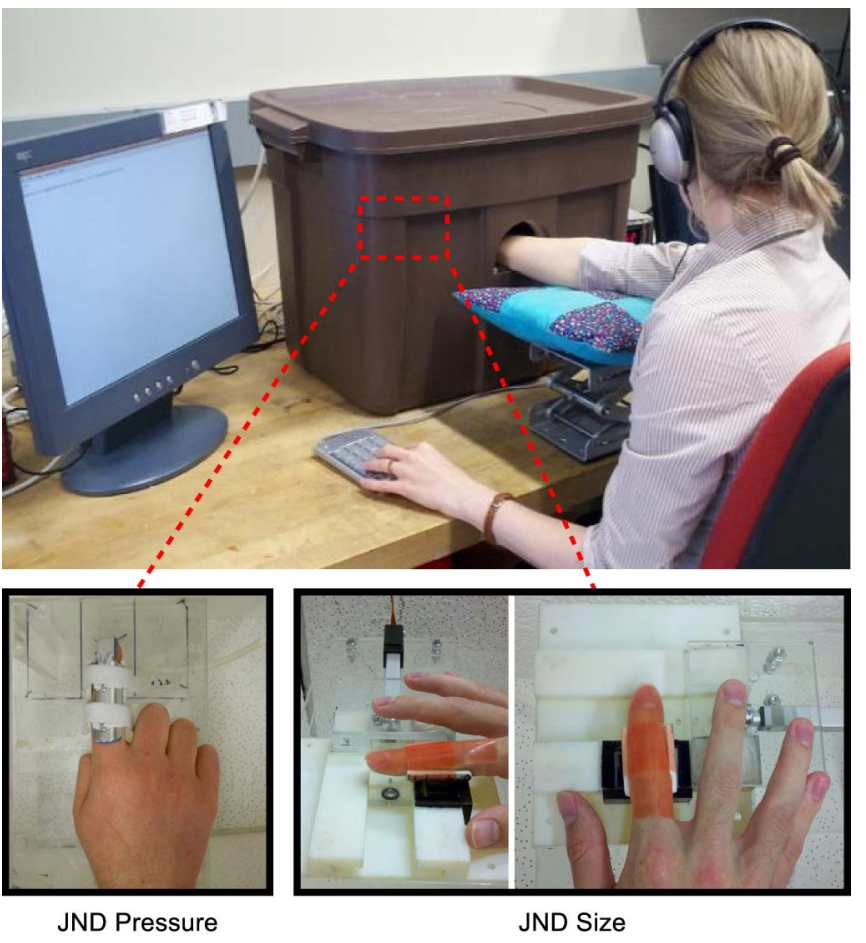

Fig. 10. Setup for JND psychophysical experiments. A subject's right index finger is fixed in place $14 \mathrm{~mm}$ above the aperture. Subjects enter responses to paired stimuli using a numerical keypad with the left hand.

always judged as less than or greater than the RS, respectively. Prior to the experiment, subjects were trained briefly using manually selected stimulus pairs. These responses were not recorded.

\subsubsection{Analysis}

A subject's response proportion $(P)$ was computed for each stimulus level and expressed as $P=\sum y_{i} / n$, where $y_{i}=1$ if the CS was perceived as greater than the RS, and $y_{i}=0$ otherwise, and $n$ is the number of pairwise discriminations performed for each CS. A psychometric function was fit to each data set using the pypsignifit toolbox version 3.0, with the default logistic function, which implements the maximum-likelihood method described by Wichmann and Hill [25], [26]. The pypsignifit toolbox was also used to evaluate the goodness of fit for each curve in terms of deviance and deviance residuals, as further described by Fründ et al. [27]. The lower and upper difference thresholds occur where the CS is judged to be greater than the RS 25 and 75 percent of the time, respectively. Each reported JND was computed by averaging the lower and upper difference thresholds.

\subsection{Pressure JND Experiment and Results}

The aim of this experiment was to determine the minimum change in air supply pressure corresponding to a JND in perceived pressure at each aperture size. The experiment consisted of five levels, one for each of the five aperture sizes tested $(1.3,1.6,1.9,2.2,2.5 \mathrm{~mm})$. The order of aperture sizes tested was randomized for each subject. Each stimulus pair consisted of a RS of 45 psig ( $310.3 \mathrm{kPag})$ and one of seven equally spaced CS ranging from 30 to 60 psig (206.8$314.7 \mathrm{kPag}$ ), separated by an ISI of $500 \mathrm{~ms}$. Each level consisted of 168 paired stimuli $(7 \mathrm{CS} \times 24$ pairwise
TABLE 4

Summary of Pressure JNDs (kPag)

\begin{tabular}{clllll}
\hline \multirow{2}{*}{ Subject } & \multicolumn{5}{c}{ Aperture Diameter } \\
\cline { 2 - 6 } & $\mathbf{1 . 3} \mathbf{~ \mathbf { m }}$ & $\mathbf{1 . 6} \mathbf{~ m m}$ & $\mathbf{1 . 9} \mathbf{~ m m}$ & $\mathbf{2 . 2} \mathbf{~ m m}$ & $\mathbf{2 . 5} \mathbf{~ m m}$ \\
\hline $\mathbf{1}$ & 24.1 & 22.3 & 21.3 & 25.1 & 19.7 \\
$\mathbf{3}$ & 15.8 & 23.1 & 16.3 & 15.2 & 14.7 \\
$\mathbf{4}$ & $25.8 *$ & 20.0 & 15.6 & 18.2 & 21.7 \\
$\mathbf{5}$ & 27.7 & 26.3 & 27.7 & 24.1 & 19.7 \\
$\mathbf{6}$ & 30.2 & 37.1 & 27.9 & 29.0 & 33.4 \\
$\mathbf{7}$ & 27.4 & 17.0 & 15.6 & 14.4 & 18.6 \\
$\mathbf{8}$ & 31.8 & 30.7 & 40.6 & 35.2 & 18.2 \\
$\mathbf{9}$ & $\mathbf{2 5 . 7}$ & 31.2 & 21.5 & 23.8 & 20.6 \\
$\mathbf{1 0}$ & 19.7 & 14.7 & 13.8 & 3.3 & 18.2 \\
\hline Mean & $\mathbf{2 4 . 4}$ & $\mathbf{1 2 . 9}$ & $* 20.2$ & 18.6 & 11.9 \\
\hline Std. Dev. & $\mathbf{5 . 7}$ & $\mathbf{7 . 8}$ & $\mathbf{8 . 6}$ & $\mathbf{8 . 9}$ & $\mathbf{5 . 6}$ \\
\hline
\end{tabular}

* Indicates JND obtained from a psychometric curve which did not demonstrate goodness of fit to the response proportion $(P)$ data. These values are excluded from mean and standard deviation calculations.

discriminations) presented randomly, and lasted approximately 15 minutes, with at least a two-minute break between levels. Subjects wore in-ear headphones playing white noise and over-ear hearing protection to prevent auditory cues (Fig. 10 (top)). An additional finger brace fastened around the back of the finger secured the finger to the holder and prevented motion of the proximal interphalangeal joint.

The pressure JND data presented here are from a preliminary study (Bianchi et al. [28]). In this work, we analyze the results using a newer pypsignifit toolbox version 3.0 for python (http://psignifit.sourceforge.net/), which are reported in Table 4 . Each numerical result is the pressure JND obtained from the psychometric function fit to that data set using the pypsignifit toolbox. JND values for pressure range from 19.6-24.4 kPag. The mean JND pressure values monotonically decrease as the aperture size increases. A multilinear regression of the mean pressure JND levels against the experiment levels returns $r^{2}=0.99$ and $p<0.001$, indicating a significant correlation between the aperture size and the pressure JND. This result is considered in more detail in the discussion.

\subsection{Aperture Size JND Experiment and Results}

The aim of this experiment was to determine the minimum change in aperture size corresponding to a JND in perceived size at a fixed peak pressure on the finger pad. Subjects were asked to perform the experiment at three separate levels, one for each selected peak pressure $(10,30,50 \mathrm{kPag})$. Each stimulus pair consisted of a RS of $2.75 \mathrm{~mm}$ and one of five equally spaced CS, which are summarized along with the supply pressures used for each level in Table 5. The ISI was slightly longer for this experiment (1.5 s) to account for motor movement between aperture sizes. Each level of the experiment consisted of 120 paired-stimulus trials (5 CS $\times 24$ pairwise discriminations) presented randomly, and lasted approximately 15 minutes. Subjects wore 32-dB reduction ear plugs and over-ear noise canceling headphones playing 
TABLE 5

Aperture Size JND Psychophysical Experiment

\begin{tabular}{|c|c|c|c|c|c|}
\hline \multirow[b]{3}{*}{ Peak Pressure (kPag) } & \multicolumn{5}{|c|}{ Aperture Diameter (mm) } \\
\hline & 1.9 & 2.3 & 2.75 & 3.2 & 3.6 \\
\hline & \multicolumn{5}{|c|}{ Supply Pressures (psig) } \\
\hline $10 \mathrm{kPag}$ & 10 & 9 & 8 & 11 & 13 \\
\hline $30 \mathrm{kPag}$ & 27 & 22 & 21 & 24 & 29 \\
\hline $50 \mathrm{kPag}$ & 44 & 36 & 32 & 35 & 39 \\
\hline
\end{tabular}

white noise to prevent auditory cues. For this experiment, the index finger was allowed to move horizontally approximately $3 \mathrm{~mm}$ in each direction, thus allowing the index finger to explore the stimulus size while maintaining a fixed stimulus-finger distance.

Since the focus of this experiment was to understand perceptual changes in the size of the stimulus, it was crucial to decouple perceived pressure and size so subjects were only making judgments about the aperture size (and not the changes in perceived pressure). This required using separately calculated supply pressure levels for each aperture size tested. The tactile measurements allow this relationship to be determined empirically because it relates the supply pressures, aperture sizes, and the resulting peak impingement pressures in the same data set. Supply pressure levels for each aperture size were calculated by selecting a constant peak pressure and interpolating to find the necessary supply pressures to achieve that particular peak pressure.

For this experiment, additions were made to the experimental apparatus described in Section 3.2. The experimental apparatus (Fig. 10 (right)) consisted of a linear actuator (Firgelli L16, Victoria, British Columbia, Canada) connected to a 5.4-mm-thick acrylic plate with the five CS aperture sizes (Table 5). The acrylic plate slides in a track along the direction of motor actuation over the air supply aperture. An O-ring seated in the base sliding track created an airtight seal between the base and the acrylic plate when the system was pressurized, forcing all supplied air through the acrylic plate aperture. A voltage signal controlled the stroke distance of the linear actuator that moved the acrylic plate between aperture sizes $\left(20 \mathrm{mms}^{-1} \max\right.$ speed $)$. Predetermined voltage values corresponding to linear actuator stroke positions allowed the acrylic plate apertures to be placed over the source aperture accurately and repeatably. Motor movement vibrations generated while moving between aperture sizes were difficult to perceive. Trials with closely spaced apertures were buffered with additional motor movement to make vibrations similar enough in duration to be safely ignored.

Aperture size JND values are obtained by the procedure described in Section 3.4.2 and are reported in Table 6. Mean values for aperture size JND range from $0.50-0.66 \mathrm{~mm}$. A multilinear regression of the mean JND values to the experiment levels returns $r^{2}=0.52$ and $p=0.49$, indicating no significant correlation between the supply pressure and the subjects' sensitivity to changes in perceived lump size.
TABLE 6

Summary of Aperture Size JNDs (mm)

\begin{tabular}{|c|c|c|c|}
\hline \multirow{2}{*}{ Subject } & \multicolumn{3}{|c|}{ Peak Pressure (kPag) } \\
\hline & $10 \mathrm{kPag}$ & $30 \mathrm{kPag}$ & $50 \mathrm{kPag}$ \\
\hline 1 & 0.36 & 0.30 & 1.23 \\
\hline 2 & 0.60 & 0.39 & 0.46 \\
\hline 3 & $1.05 *$ & $1.74 *$ & 0.76 \\
\hline 4 & $0.43 *$ & 0.85 & 0.51 \\
\hline 5 & 0.56 & 0.33 & $0.33 *$ \\
\hline 6 & 0.37 & 0.31 & 0.92 \\
\hline 7 & 0.74 & $0.43 *$ & 0.70 \\
\hline 8 & $0.61 *$ & 0.66 & $0.71 *$ \\
\hline 9 & 1.16 & 0.62 & 0.79 \\
\hline 10 & 0.24 & 0.12 & 0.26 \\
\hline 11 & 0.19 & 0.22 & 0.24 \\
\hline 12 & 0.71 & 0.43 & 0.60 \\
\hline 13 & $0.38 *$ & $0.03 *$ & 0.43 \\
\hline 14 & $1.19 *$ & 1.23 & 0.83 \\
\hline 15 & $0.83 *$ & 0.51 & $0.61 *$ \\
\hline 16 & 0.45 & $0.14 *$ & 0.82 \\
\hline Mean & 0.54 & 0.50 & 0.66 \\
\hline Std. Dev. & 0.29 & 0.31 & 0.28 \\
\hline
\end{tabular}

* Indicates JND obtained from a psychometric curve which did not demonstrate goodness of fit to the response proportion $(P)$ data. These values are excluded from mean and standard deviation calculations.

\section{Discussion}

\subsection{Air-Jet Design and Characterization}

\subsubsection{Air-Jet Impingement Surface}

A significant body of literature describes jet impingement on a hard, flat surface or plate. In this work, the tactile sensor array serves as a flat impinging surface, making these prior results a reasonable reference for comparison to the data obtained in this study. However, the intended target of the pneumatic lump display is the human finger, which is (by comparison) not firm or flat, but exhibits compliance and curvature that change the local shape of the finger as it is stimulated. While the tactile sensor array characterizes the display output and captures the isolated effects of the control parameters upon the output, the psychophysical studies implicitly account for complex factors such as skin compliance, finger curvature, and perceptual capabilities as a function of the stimulus parameters.

\subsubsection{Jet Spread Rate}

For approximately $H / D>5$, the jet expansion rate is effectively linear $\left(r^{2}=0.99\right)$, consistent with jet theory [24]. Fig. 8 shows that the ratio $b / H=0.09$, which is comparable to the values obtained by Hussein et al. (0.096) [29] and Panchapakesan et al. (0.102, 0.094) [30], [31]. An important consideration in this comparison to previous work is that the jet spread rate, $S$, was defined in earlier studies by the radial width of the profile at half of the centerline velocity. Since velocity in the jet flow was not measured in this study, the width of the Gaussian pressure profile at half of the maximum impingement pressure was used instead. Further comparisons of this width metric to 
data from $\mathrm{Tu}$ and Wood [24] show that the air-jet lump display expands in a comparable manner over a similar range of dimensionless impingement ratios (Fig. 8).

\subsubsection{Implications for Air-Jet Lump Display Control}

Results from Fig. 6 have important implications for the control of an air-jet lump display mechanism. Fig. 6a indicates that the amplitude, or desired peak impingement pressure $(\alpha)$, of the display is affected by both the aperture size and the supply pressure. The curves on this plot represent the "display-space" of the mechanism given the current range of parameters and describes the space of achievable peak impingement pressures. Changes in peak impingement pressure are most sensitive to changes in aperture size at larger supply pressures. In contrast, Fig. 6b shows that the width of the pressure profile $(\sigma)$ is largely independent of supply pressure and is primarily a linear function of the aperture size. If psychophysical perception of lump size is indeed dictated by the width of the Gaussian pressure profile, then this result suggests that perceived lump size could be controlled only by adjusting the aperture size.

\subsubsection{Potential for Use in Surgery}

The air-jet lump display described in this work only requires a regulated air supply line and a simple mechanism to change aperture size. One such mechanism was investigated by the authors previously [32], in which various apertures were placed circumferentially around a disk that quickly rotated over a central supply source to dynamically change the effective aperture size. This prototype demonstrated that the control principles of the air-jet lump display functioned effectively even when encased in a compact package. Other studies have demonstrated that novel devices can be made small enough to approach the size constraints imposed by RMIS integration [17].

While the experiments in this work are limited to constant stimuli, the transient characteristics of the display are also important for a lump display in RMIS palpation, where the pressure profile produced upon the finger should change dynamically during palpation. The prototype in [32] demonstrated that the air pressure and aperture size can be dynamically changed together or independent of the opposite control parameter. Through informal testing, we found that the percepts generated on the finger are noticeably different as the stimulus control parameters are changed.

\subsection{Psychophysical Studies}

\subsubsection{Effects of Temperature on Tactile Discrimination}

In both the JND pressure and aperture size experiments, some subjects commented that their stimulated finger pads began to feel cold in the latter stages of the experiment. This is due to the increased rate of convective heat transfer from the finger to the ambient air as well as an increased rate of moisture evaporation from the skin. Previous research suggests that different populations of mechanoreceptors may differ in their susceptibility to thermal influence. For finger skin temperatures above $8^{\circ} \mathrm{C}$, spatial and pressure

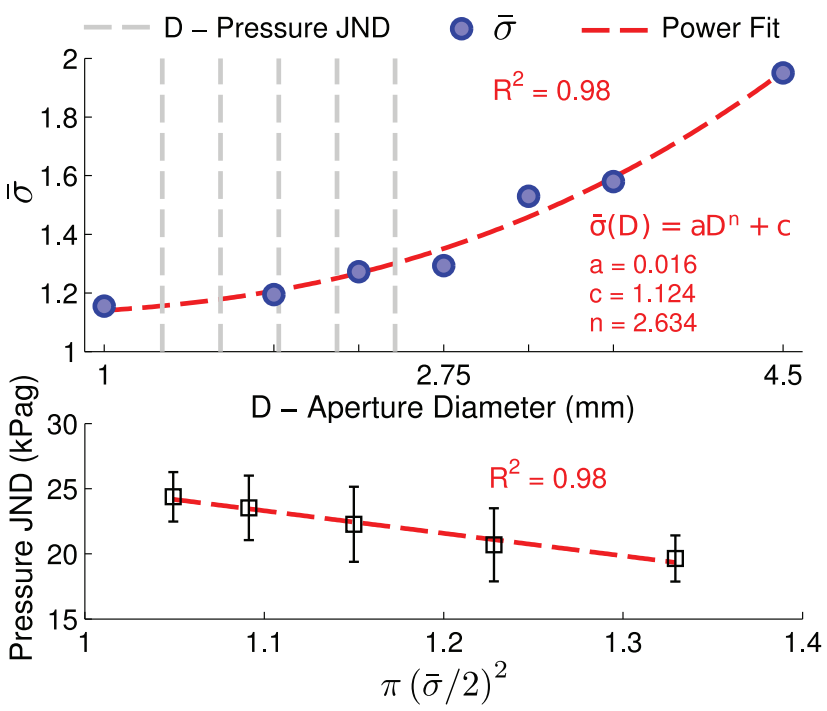

Fig. 11. (Top) The average $\sigma$ value $(\bar{\sigma})$ for each aperture size in the tactile sensor data is fit with a power function. $\bar{\sigma}$ for pressure JND values (denoted by vertical gray lines) are interpolated from the resulting fit equation. (Bottom) A linear correlation $\left(r^{2}=0.98\right)$ is observed between the mean pressure JND values and the estimated jet contact area.

discrimination remain relatively unaffected, although below this temperature deterioration occurs rapidly [33], [34]. In contrast, cooler temperatures impair sensitivity to highfrequency vibration by decreasing sensitivity of Pacinian corpuscles, especially for stimulus frequency components greater than approximately $80 \mathrm{~Hz}$ [35]. Since this study did not directly evaluate thermal effects of air-jet stimulus perception, it is difficult to know to what extent thermal effects influenced experimental results. However, to prevent or minimize these types of effects, both psychophysics studies were broken into short blocks with subjects taking breaks in between blocks. Additionally, the order in which different experiment levels were performed was randomized, so that potential effects of finger skin temperature on tactile discrimination were minimized.

\subsubsection{Contact Area and Pressure Discriminability}

Results from the pressure JND study revealed a significant correlation $\left(r^{2}=0.99\right.$ and $\left.p<0.001\right)$ between decreasing pressure JND and increasing aperture size. One explanation may be the size of the contact area that was stimulated by the air jet during discrimination. The mean spatial spread $(\bar{\sigma})$ of the pressure profile curves at each aperture size used in the tactile sensor measurements was calculated (Fig. 11 (top)), then prescribed a power function fit $\left(r^{2}=0.98\right)$. Values of $\bar{\sigma}$ for JND pressure aperture sizes were interpolated using the resulting fit equation.

As aperture size increases, the effective jet area upon the finger increases in proportion to $\pi(\bar{\sigma} / 2)^{2}$. Therefore, larger aperture sizes produce an air jet with a larger impingement area on the finger pad, resulting in a larger number of mechanoreceptors being used for discrimination. A linear relationship $\left(r^{2}=0.98\right)$ exists between jet area and the mean pressure JND values (Fig. 11 (bottom)), supporting the notion that experiencing the air-jet stimulus over a larger region of the skin (and mechanoreceptors) makes differential discrimination easier. 


\subsubsection{Comparison to Known Human Limits}

Tactile spatial resolution in the human fingerpad under conditions of static touch (i.e., without lateral scanning) is about $1 \mathrm{~mm}$ [36], which corresponds closely to the distance between sensory receptor units at the fingertips. In the JND size experiment, subjects were allowed to scan their finger laterally with respect to the stimulus, which dynamically deforms the skin during movement. It is known that lateral scanning can improve tactile performance [37], which may explain why our JND values $(0.5-0.66 \mathrm{~mm})$ are less than $1 \mathrm{~mm}$. For additional comparison, Grant et al. [38] showed that the threshold for Braille dot discrimination was $0.58 \mathrm{~mm}$, which compares favorably with our results.

\section{Conclusions}

An air-jet lump display is a promising technique to elicit the percept of feeling a lump with the bare finger. This display type is simple in design and can display a wide variety of lump-like pressure profiles to the finger by changing two control parameters (air supply pressure and aperture size). The quantitative results indicate that peak impingement pressure $(\alpha)$ is dependent on both supply pressure and aperture size, while the pressure profile width $(\sigma)$ is solely dependent on the aperture size.

The psychophysical studies reveal that perceivable changes in air jet aperture size (diameter) are on the order of $\sim 0.5-0.65 \mathrm{~mm}$ and are unaffected by the supply pressure. In contrast, perceivable changes in air-jet pressure range from $\sim 20-25 \mathrm{kPag}$, and are inversely correlated with the airjet aperture diameter and the resulting jet impingement contact area. The psychophysical JND values provide a benchmark for further development of tactile displays utilizing air-jet-based mechanisms, or other haptic technology using air jets. Furthermore, the thorough characterization of a single air jet as a haptic stimulus can improve future design and development of displays that use multiple air jets in an array (or other pattern) to produce more elaborate haptic stimuli, and stimulate regions of the skin larger than a single finger pad.

\section{ACKNOWLEDGMENTS}

This work was supported in part by the US Army Medical Research and Material Command under contract no. W81XWH-11-C-0050, a National Science Foundation Graduate Research Fellowship, and the European Commission Collaborative Project no. 248587, The Hand Embodied, within the FP7-ICT-2009-4-2-1 Cognitive Systems and Robotics Program.

\section{REFERENCES}

[1] A.R. Lanfranco, A.E. Castellanos, J.P. Desai, and W.C. Meyers, "Robotic Surgery: A Current Perspective," Annals of Surgery, vol. 239, no. 1, pp. 14-21, 2004.

[2] E.P. Westebring-van der Putten, R.H.M. Goossens, J.J. Jakimowicz, and J. Dankelman, "Haptics in Minimally Invasive Surgery-A Review," Minimally Invasive Therapy and Allied Technologies, vol. 17, no. 1, pp. 3-16, 2008.

[3] G. Tholey, J.P. Desai, and A.E. Castellanos, "Force Feedback Plays a Significant Role in Minimally Invasive Surgery: Results and Analysis," Annals of Surgery, vol. 241, no. 1, pp. 102-109, 2005.
[4] C. Wagner and R. Howe, "Force Feedback Benefit Depends on Experience in Multiple Degree of Freedom Robotic Surgery Task," IEEE Trans. Robotics, vol. 23, no. 6, pp. 1235-1240, Dec. 2007.

[5] S. Schostek, M.O. Schurr, and G.F. Buess, "Review on Aspects of Artificial Tactile Feedback in Laparoscopic Surgery," Medical Eng. and Physics, vol. 31, no. 8, pp. 887-98, 2009.

[6] K. Hoyt, B. Castaneda, M. Zhang, P. Nigwekar, P.A. di Sant'agnese, J.V. Joseph, J. Strang, D.J. Rubens, and K.J. Parker, "Tissue Elasticity Properties as Biomarkers for Prostate Cancer," Cancer Biomarkers, vol. 4, nos. 4/5, pp. 213-25, 2008.

[7] M. Zhang, P. Nigwekar, B. Castaneda, K. Hoyt, J.V. Joseph, A. di Sant'agnese, E.M. Messing, J.G. Strang, D.J. Rubens, and K.J. Parker, "Quantitative Characterization of Viscoelastic Properties of Human Prostate Correlated with Histology," Ultrasound in Medicine and Biology, vol. 34, no. 7, pp. 1033-1042, 2008.

[8] M.V. Ottermo, M.M. Ovstedal, T. Lango, O. Stavdahl, Y. Yavuz, T.A. Johansen, and R. Marvik, "The Role of Tactile Feedback in Laparoscopic Surgery," Surgical Laparoscopy Endoscopy and Percutaneous Techniques, vol. 16, no. 6, pp. 390-400, 2006.

[9] D.M. Ota, "Laparoscopic Colectomy for Cancer: A Favorable Opinion," Annals of Surgical Oncology, vol. 2, no. 1, pp. 3-5, 1995.

[10] M.V. Ottermo, O. Stavdahl, and T.A. Johansen, "A Remote Palpation Instrument for Laparoscopic Surgery: Design and Performance," Minimally Invasive Therapy and Allied Technologies, vol. 18, no. 5, pp. 259-272, 2009.

[11] R. Howe, W. Peine, D. Kantarinis, and J. Son, "Remote Palpation Technology," IEEE Eng. Medicine and Biology Magazine, vol. 14, no. 3, pp. 318-323, May/June 1995.

[12] S. Kim, K. Kyung, J. Park, and D. Kwon, "Real-Time Area-Based Haptic Rendering and the Augmented Tactile Display Device for a Palpation Simulator," Advanced Robotics, vol. 21, no. 9, pp. 961-981, 2007.

[13] J. Killebrew, S. Bensmaia, J. Dammann, P. Denchev, S. Hsiao, J. Craig, and K. Johnson, "A Dense Array Stimulator to Generate Arbitrary Spatio-Temporal Tactile Stimuli," J. Neuroscience Methods, vol. 161, no. 1, pp. 62-74, 2007.

[14] A.M. Okamura, "Haptic Feedback in Robot-Assisted Minimally Invasive Surgery," Current Opinion in Urology, vol. 19, pp. 102-107, 2009.

[15] I. Sarakoglou, N.G. Tsagarakis, and D.G. Caldwell, "A Compact Tactile Display Suitable for Integration in VR and Teleoperation," Proc. IEEE Int'l Conf. Robotics and Automation, pp. 1018-1024, 2012.

[16] Y. Kim, I. Oakley, and J. Ryu, "Human Perception of Pneumatic Tactile Cues," Advanced Robotics, vol. 22, no. 8, pp. 807-828, 2008.

[17] C.-H. King, M.O. Culjat, M.L. Franco, J.W. Bisley, E. Dutson, and W.S. Grundfest, "Optimization of a Pneumatic Balloon Tactile Display for Robot-Assisted Surgery Based on Human Perception," IEEE Trans. Biomedical Eng., vol. 55, no. 11, pp. 2593-2600, Nov. 2008.

[18] L. Santos-Carreras, K. Leuenberger, P. Retornaz, R. Gassert, and H. Bleuler, "Design and Psychophysical Evaluation of a Tactile Pulse Display for Teleoperated Artery Palpation," Proc. IEEE Int'l Conf. Intelligent Robots and Systems, pp. 5060-5066, 2010.

[19] M. Cohn, "Tactile Feedback for Teleoperation," Proc. SPIE, vol. 1833, pp. 240-254, 1992.

[20] G. Moy, C. Wagner, and R. Fearing, "A Compliant Tactile Display for Teletaction," Proc. IEEE Int'l Conf. Robotics and Automation, pp. 3409-3415, 2000.

[21] K. Inoue, F. Kato, and S. Lee, "Haptic Device Using Flexible Sheet and Air Jet for Presenting Virtual Lumps under Skin," Proc. IEEE Int'l Conf. Intelligent Robots and Systems, pp. 1749-1754, 2009.

[22] T. Davanipour and S. Sami, "Short Jet Impingement," J. Hydraulics Division, vol. 103, no. 5, pp. 557-567, 1977.

[23] S. Pope, Turbulent Flows. Cambridge Univ. Press, 2000

[24] C.V. Tu and D.H. Wood, "Wall Pressure and Shear Stress Measurements Beneath an Impinging Jet," Experimental Thermal and Fluid Science, vol. 13, no. 4, pp. 364-373, 1996.

[25] F. Wichmann and N. Hill, "The Psychometric Function: I. Fitting, Sampling, and Goodness of Fit," Perception and Psychophysics, vol. 63 , no. 8 , pp. $1293-1313,2001$

[26] F. Wichmann and N. Hill, "The Psychometric Function: II. Bootstrap-Based Confidence Intervals and Sampling," Perception and Psychophysics, vol. 63, no. 8, pp. 1314-1329, 2001.

[27] I. Fründ, N.V. Haenel, and F.A. Wichmann, "Inference for Psychometric Functions in the Presence of Nonstationary Behavior," J. Vision, vol. 11, no. 6, pp. 1-19, 2011. 
[28] M. Bianchi, J.C. Gwilliam, A. Degirmenci, and A.M. Okamura, "Characterization of an Air Jet Haptic Lump Display," Proc. IEEE Conf. Eng. Medicine and Biology Soc., pp. 3467-3470, 2011.

[29] H. Hussein, S. Capp, and W. George, "Velocity Measurements in a High-Reynolds-Number, Momentum-Conserving, Axisymmetric, Turbulent Jet," J. Fluid Mechanics, vol. 258, no. 1, pp. 31-75, 1994.

[30] N. Panchapakesan and J. Lumley, "Turbulence Measurements in Axisymmetric Jets of Air and Helium. Part 1. Air Jet," J. Fluid Mechanics, vol. 246, no. 1, pp. 197-223, 1993

[31] N. Panchapakesan and J. Lumley, "Turbulence Measurements in Axisymmetric Jets of Air and Helium. Part 2. Helium Jet," J. Fluid Mechanics, vol. 246, no. 1, pp. 225-247, 1993.

[32] J.C. Gwilliam, A. Degirmenci, M. Bianchi, and A.M. Okamura, "Design and Control of an Air-Jet Lump Display," Proc. IEEE Haptics Symp., pp. 45-49, 2012.

[33] K. Provins and R. Morton, "Tactile Discrimination and Skin Temperature," J. Applied Physiology, vol. 15, pp. 155-160, 1960.

[34] J. Stevens, B. Green, and A. Krimsley, "Punctate Pressure Sensitivity: Effects of Skin Temperature," Sensory Processes, vol. 1, no. 3, pp. 238-243, 1977.

[35] B. Green, "The Effect of Skin Temperature on Vibrotactile Sensitivity," Attention, Perception, and Psychophysics, vol. 21, no. 3, pp. 243-248, 1977.

[36] R.W. Van Boven and K.O. Johnson, "The Limit of Tactile Spatial Resolution in Humans: Grating Orientation Discrimination at the Lip, Tongue, and Finger," Neurology, vol. 44, no. 12, pp. 2361-2361, 1994.

[37] J. Morley, A. Goodwin, and I. Darian-Smith, "Tactile Discrimination of Gratings," Experimental Brain Research, vol. 49, no. 2, pp. 291-299, 1983.

[38] A.C. Grant, M.C. Thiagarajah, and K. Sathian, "Tactile Perception in Blind Braille Readers: A Psychophysical Study of Acuity and Hyperacuity Using Gratings and Dot Patterns," Perception and Psychophysics, vol. 62, no. 2, pp. 301-312, 2000.

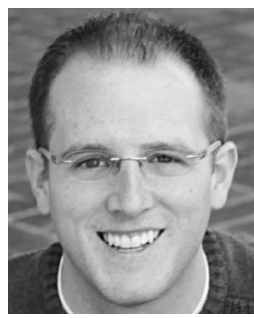

James C. Gwilliam received the BS degree in biomedical engineering from the University of Utah in 2007. He is currently working toward the $\mathrm{PhD}$ degree in biomedical engineering at The Johns Hopkins University School of Medicine and is a visiting graduate researcher in the Department of Mechanical Engineering at Stanford University, California. His research interests include medical robotics, tactile feedback in robot-assisted surgery, and novel tactile display mechanisms. He received the Graduate Research Fellowship from the US National Science Foundation in 2009. He is a student member of the IEEE.

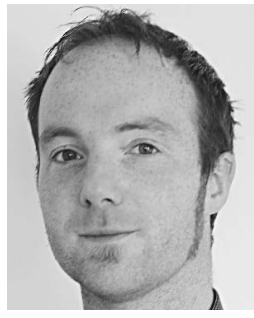

Matteo Bianchi received the BS degree and the MS degree in biomedical engineering and the $\mathrm{PhD}$ degree in automatics, robotics, and bioengineering from the University of Pisa, Italy, in 2004, 2007, and 2012, respectively. $\mathrm{He}$ is currently a postdoctoral researcher at the Interdepartmental Research Center "E. Piaggio" of the University of Pisa. From January 2011 through June 2011, he was a visiting student at the Laboratory for Computational Sensing and Robotics, The Johns Hopkins University, Baltimore, Maryland. His research interests include haptic interfaces, medical robotics, tactile interaction modeling, psychophysics of the sense of touch and human manipulation, optimal hand pose sensing, and tracking. He has authored contributions to international conferences and journals. He is a member of the IEEE.

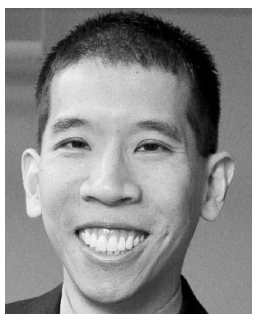

Lester $\mathbf{K}$. Su received the $A B$ degree in physics from the University of Chicago, Illinois, in 1990 and the $\mathrm{PhD}$ degree in aerospace engineering from the University of Michigan, Ann Arbor, in 1995. He also received the MS degree in mathematics and the MSE degree in aerospace engineering from the University of Michigan. He is currently a consulting associate professor of mechanical engineering at Stanford University. He was previously an assistant professor of mechanical engineering at The Johns Hopkins University. His research interests include laser imaging methods for fluid mechanics and combustion, modeling approaches for simulations of turbulent flows, multiphase flow dynamics, and hydrodynamic propulsion mechanisms. He received the 2004 US National Science Foundation CAREER Award and several teaching and mentoring awards from The Johns Hopkins University, including the Alumni Association Excellence in Teaching Award, the George E. Owen Teaching Award, the W.H. Huggins Excellence in Teaching Award, the McDonald Award for Excellence in Mentoring and Advising, and the Diversity Recognition Award for his work in engineering outreach.

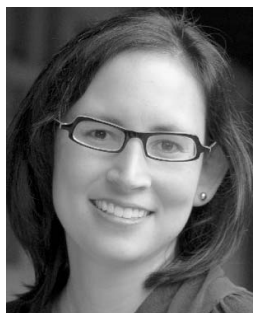

Allison M. Okamura received the BS degree from the University of California, Berkeley, in 1994, and the MS and PhD degrees from Stanford University, California, in 1996 and 2000 , respectively, all in mechanical engineering. She is currently an associate professor of mechanical engineering at Stanford University, California, where she is also the Robert Bosch Faculty Scholar. She was previously a professor and the vice chair of mechanical engineering at The Johns Hopkins University, Baltimore, Maryland. Her research interests include haptics, teleoperation, medical robotics, virtual environments and simulation, neuromechanics and rehabilitation, prosthetics, and engineering education. She received the 2004 US National Science Foundation CAREER Award, the 2005 IEEE Robotics and Automation Society Early Academic Career Award, and the 2009 IEEE Technical Committee on Haptics Early Career Award. She is a fellow of the IEEE and an associate editor of the IEEE Transactions on Haptics.

For more information on this or any other computing topic, please visit our Digital Library at www.computer.org/publications/dlib. 